\title{
Multimedia Security Application of a Ten-Term Chaotic System without Equilibrium
}

\author{
Xiong Wang, ${ }^{1}$ Akif Akgul, ${ }^{2}$ Sezgin Kacar, ${ }^{2}$ and Viet-Thanh Pham ${ }^{3}$ \\ ${ }^{1}$ Institute for Advanced Study, Shenzhen University, Shenzhen, Guangdong 518060, China \\ ${ }^{2}$ Department of Electrical and Electronics Engineering, Faculty of Technology, Sakarya University, Serdivan, Turkey \\ ${ }^{3}$ Modeling Evolutionary Algorithms Simulation and Artificial Intelligent, Faculty of Electrical \& Electronics Engineering, \\ Ton Duc Thang University, Ho Chi Minh City, Vietnam
}

Correspondence should be addressed to Viet-Thanh Pham; phamvietthanh@tdt.edu.vn

Received 19 July 2017; Revised 4 October 2017; Accepted 31 October 2017; Published 15 November 2017

Academic Editor: Dimitri Volchenkov

Copyright (C) 2017 Xiong Wang et al. This is an open access article distributed under the Creative Commons Attribution License, which permits unrestricted use, distribution, and reproduction in any medium, provided the original work is properly cited.

A system without equilibrium has been proposed in this work. Although there is an absence of equilibrium points, the system displays chaos, which has been confirmed by phase portraits and Lyapunov exponents. The system is realized on an electronic card, which exhibits chaotic signals. Furthermore, chaotic property of the system is applied in multimedia security such as image encryption and sound steganography.

\section{Introduction}

Recently, there is an increased interest in multimedia communication, which is vital in various areas ranging from entertainment industries, economics, and medical applications to militaries $[1,2]$. Several attempts have been made to provide confidentiality, identity, and integrity. A considerable amount of studies about multimedia security has been introduced, for example, data encryption, steganographic messages, watermarking, or multimedia authentication [1-6].

Extensive researches have shown that the use of chaos for multimedia communication is a potential application [711]. Because of the similar characteristics between chaotic systems and cryptographic primitives, chaotic cryptography is a major area of interest [12]. Liu et al. presented the audio encryption scheme with confusion and diffusion [13]. They based on a multiscroll chaotic system and one-time keys [13]. By using complex hyperchaotic systems, color image encryption was proposed [14]. Chaos maps with Markov properties were applied to construct a new encryption algorithm [15]. In addition, Ghebleh and Kanso introduced a robust chaos scheme to image steganography [16]. In order to generate message authentication codes, suitability of chaos functions was studied in [17].
In this work, we study a ten-term chaotic system without equilibrium and its multimedia security application. The chaotic attractors in this system are "hidden attractors" because the basin of attraction for a hidden attractor is not connected with any unstable fixed point [18-21]. It is noted that the concept of hidden attractors has been suggested in connection with the occurrence of unpredictable attractors in multistable systems [21]. Researchers have shown that multistability is connected with the occurrence of unpredictable attractors [21-30]. Recently, hidden attractor has been investigated in numerous systems such as Chua system [19], drilling system [31], Lorenz-like system [32], Goodwin oscillator [33], electromechanical systems [34], twodimensional maps [35], phase-locked loop circuits [36], and Rabinovich-Fabrikant system [37].

\section{A System without Equilibrium and Its Implementation}

2.1. A System without Equilibrium. Chaotic systems without equilibrium attract have been attracting a lot of interest [3842]. When mentioning a chaotic system without equilibrium, it means that there is no real equilibrium in such a system. 
In this work, we study a general three-dimensional form described by

$$
\begin{aligned}
& \dot{x}=a_{1} y, \\
& \dot{y}=a_{2} x+a_{3} y+a_{4} z+a_{5} x z, \\
& \dot{z}=a_{6} x+a_{7} z+a_{8} x y+a_{9} x z+a_{10},
\end{aligned}
$$

in which $a_{i}(i=1, \ldots, 10)$ are parameters and $a_{i} \neq 0$.

We can solve the following three equations to find the system's equilibrium:

$$
\begin{aligned}
a_{1} y & =0, \\
a_{2} x+a_{3} y+a_{4} z+a_{5} x z & =0, \\
a_{6} x+a_{7} z+a_{8} x y+a_{9} x z+a_{10} & =0 .
\end{aligned}
$$

From (2), we have

$$
A x^{2}+B x+C=0
$$

in which

$$
\begin{aligned}
& A=a_{2} a_{5} a_{9}-a_{5}^{2} a_{6}, \\
& B=a_{2} a_{5} a_{7}-a_{4} a_{5} a_{6}-a_{5}^{2} a_{10}, \\
& C=-a_{4} a_{5} a_{10},
\end{aligned}
$$

for

$$
a_{5} a_{7}-a_{4} a_{9} \neq 0
$$

As a result, there is no equilibrium in general form (1) for

$$
\Delta=B^{2}-4 A C<0 .
$$

Based on conditions (5) and (6) and $a_{i} \neq 0$, we have applied a search procedure [40] to find the set of parameters for which general form (1) does not have equilibrium. A set of parameters has been found as follows:

$$
\begin{aligned}
& a_{1}=a, \\
& a_{2}=a_{7}=a_{8}=-1, \\
& a_{3}=b, \\
& a_{4}=c, \\
& a_{5}=a_{6}=a_{10}=1, \\
& a_{9}=d,
\end{aligned}
$$

where $a, b, c, d>0$. General form (1) is rewritten by

$$
\begin{aligned}
& \dot{x}=a y, \\
& \dot{y}=-x+b y+c z+x z, \\
& \dot{z}=x-z-x y+d x z+1 .
\end{aligned}
$$

It is simple to see that system (8) has no equilibrium for $a=2, b=0.1, c=2.5$, and $d=0.3$. However, it displays chaos as shown in Figure 1. In this case, calculated Lyapunov exponents of the system are illustrated in Figure 2. Lyapunov exponents are $L_{1}=0.2563, L_{2}=0$, and $L_{3}=-0.5762$ for $t=10,000$. Positive Lyapunov exponent $L_{1}=0.2563$ verifies the chaotic behavior of system (8). Attractors of system (8) are hidden attractors according the introduced definition in [21]. It is noted that the hidden attractors are observed in systems without equilibria, with no unstable fixed points or with one stable fixed point [21].

2.2. Electronic Circuit Implementation of the Chaotic System without Equilibrium. Electronic implementation of a chaotic model is useful for confirming the model's feasibility as well as realizing applications [43-45]. The main aim of this section is to design a circuit to realize the system without equilibrium by using electronic elements [46-51]. The chaotic system must be rescaled for electronic circuit implementation. The amplitude values of system $(x, y$, and $z)$ are in the interval of $(-80,60)$. They are higher than the interval of $(-15,15)$ which are electronic materials limitations. For scale process, let $X / 2=x, Y / 6=y$, and $Z=z / 4$ and then adjust the original state variables $x, y, z$ instead of the variables $X, Y, Z$. As a result, the scaled system finally becomes of the following form:

$$
\begin{aligned}
& \dot{X}=3 a Y, \\
& \dot{Y}=-\frac{1}{3} X+b Y+\frac{2}{3} c Z+\frac{4}{3} X Z, \\
& \dot{Z}=\frac{1}{2} X-Z-3 X Y+2 d X Z+\frac{1}{4} .
\end{aligned}
$$

An electronic circuit is designed for the scaled system as shown in Figure 3.

As can be seen in Figure 3, we selected $C 1=C 2=C 3=$ $1 \mathrm{nF}, R 1=66 \mathrm{k} \Omega, R 2=R 3=R 8=R 9=R 15=R 16=100 \mathrm{k} \Omega$, $R 4=1200 \mathrm{k} \Omega, R 5=4000 \mathrm{k} \Omega, R 6=240 \mathrm{k} \Omega, R 7=30 \mathrm{k} \Omega, R 10=$ $800 \mathrm{k} \Omega, R 11=400 \mathrm{k} \Omega, R 12=24000 \mathrm{k} \Omega, R 13=67 \mathrm{k} \Omega$, and $R 14$ $=14 \mathrm{k} \Omega$. The scaled chaotic system was done on the electronic card (see Figure 4). All phase portraits on the oscilloscope are shown in Figure 5, which illustrate the feasibility of the system.

\section{Image Encryption and Sound Steganography Applications of the System without Equilibrium}

After considering the circuit implementation of the system, in this section, image encryption and hiding of encrypted image data in a sound file have been implemented to show that the no-equilibrium chaotic system can be used in multimedia security applications. In order to realize these applications, firstly random number generator design has been done.

3.1. Random Number Generator (RNG) Design. One of the most basic structures used in chaos-based encryption and stenography applications is RNG. In this study, before the security applications, a RNG design has been implemented 


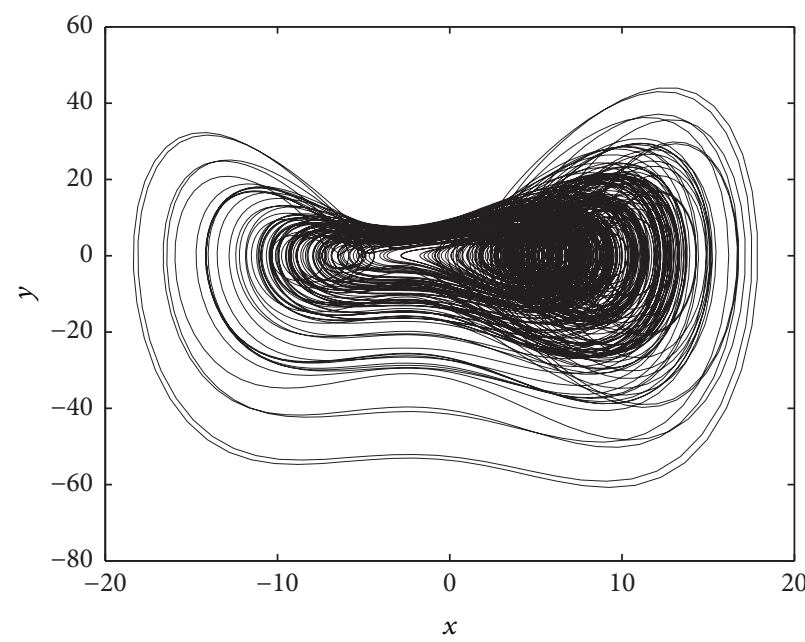

(a)

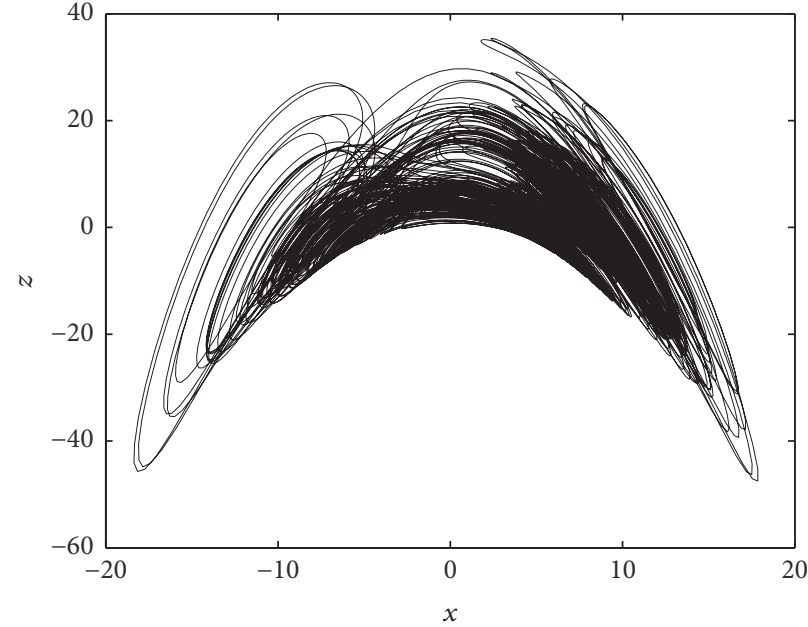

(b)

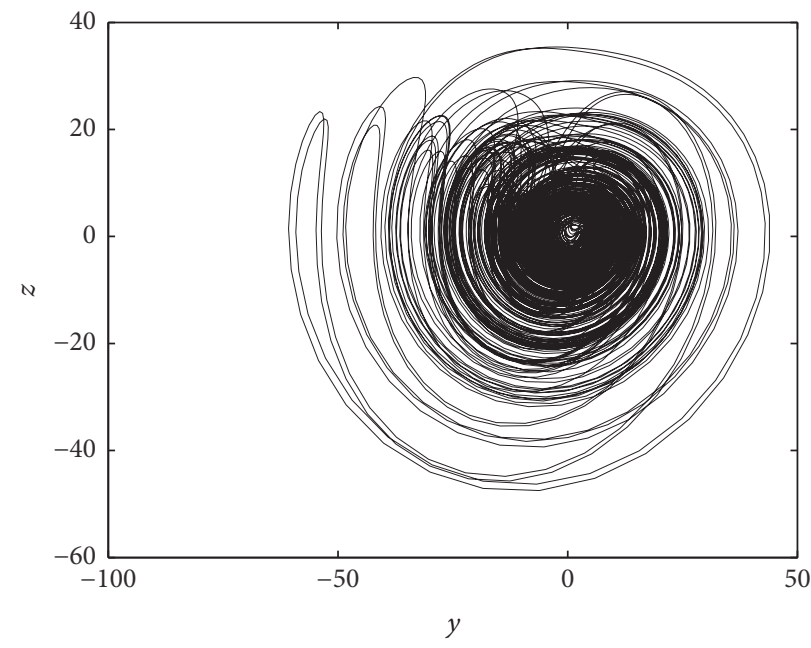

(c)

Figure 1: Projections of attractors without equilibrium in (a) $x-y$ plane, (b) $x-z$ plane, and (c) $y-z$ plane for $a=2, b=0.1, c=2.5$, and $d=0.3$ and initial conditions $(x(0), y(0), z(0))=(1,1,1)$. The attractor of system $(8)$ is "hidden" according to the definition introduced in [21].

for use in these applications. In RNG design, $\bmod (256)$ operation has been performed by taking 13, 14, and 15 digits after the point of state variables obtained by using Runge-Kutta 4 (RK4) algorithm. In this case, 8 bits of each state variable are obtained in each iteration of the RK4 algorithm. Finally, each 8-bit sequence obtained from 3 state variables $(x, y, z)$ has been XORed at each iteration to obtain a random bit sequence of RNG. In Table 1, the statistical NIST-800-22 test results of the random number sequence of $1,000,000$ bits obtained from the designed RNG are shown.

The statistical NIST-800-22 test is known as the internationally accepted best random test. The NIST test is a comprehensive test consisting of 15 different tests. In order to be able to speak of a complete randomness, obtained $P$ values from the all of 15 tests must be provided with the condition of $1>P$ value $>0.01$. When the values in Table 1 are examined, it is seen that the RNG based on the new system is successful in all tests.

3.2. Image Encryption Application. In this application, image encryption is performed using RNG obtained in Section 3.1. In the application $128 \times 128$ pixel size image with gray scale ("pepper") is used. All pixels of the original image have converted to binary and all binary bits have been subjected to $\mathrm{XOR}$ processing with a random bit sequence obtained from the RNG to perform encryption. The decryption process is also performed by applying the reverse of the encryption process. Figure 6 shows the original, encrypted, and decrypted images. As seen in the figure, the encrypted image is not understood. So, the encryption is visually very successful. Correlation, histogram, entropy, and differential attack analyses were also performed to analyze the performance of the 
TABLE 1: NIST-800-22 test results of the new chaotic system based RNG.

\begin{tabular}{lcr}
\hline Statistical tests & $P$ value & Result \\
\hline Frequency (Monobit) Test & 0,4556674150378 & Successful \\
Block-Frequency Test & 0,312738896039824 & Successful \\
Cumulative-Sums Test & 0,5441395972238 & Successful \\
Runs Test & 0,117478755093071 & Successful \\
Longest-Run Test & 0,88635602631487 & Successful \\
Binary Matrix Rank Test & 0,594206193094231 & Successful \\
Discrete Fourier Transform Test & 0,783086624373691 & Successful \\
Nonoverlapping Templates Test & 0,0586441453317821 & Successful \\
Overlapping Templates Test & 0,868314176679646 & Successful \\
Maurer's Universal Statistical Test & 0,349319372138117 & Successful \\
Approximate Entropy Test & 0,0419351614775444 & Successful \\
Random-Excursions Test $(x=-4)$ & 0,746783846542712 & Successful \\
Random-Excursions Variant Test $(x=-9)$ & 0,810174242469133 & Successful \\
Serial Test-1 & 0,366073450623053 & Successful \\
Serial Test-2 & 0,333453364381209 & Successful \\
Linear-Complexity Test & 0,992656091838689 & Successful \\
\hline
\end{tabular}

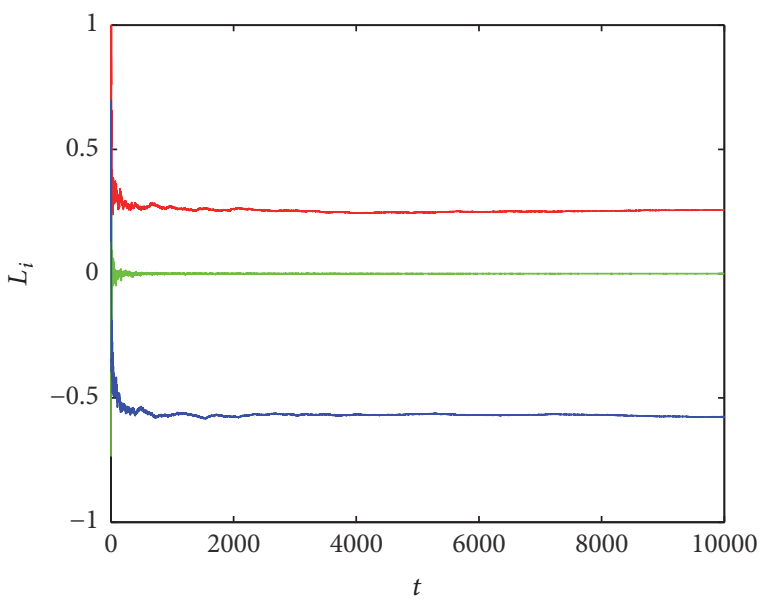

FIGURE 2: Lyapunov exponents of system without equilibrium (8) for $a=2, b=0.1, c=2.5$, and $d=0.3$ and initial conditions $(x(0), y(0), z(0))=(1,1,1): L_{1}$ (red), $L_{2}$ (green), and $L_{3}$ (blue). Positive value of Lyapunov exponent $L_{1}$ indicates chaos of system (8).

encryption. Figures 7 and 8 show correlation distributions and histogram graphs. In these graphs, it is seen that encryption process provides a very homogeneous distribution. The homogeneity of the encrypted image distributions shows the encryption is good. In addition, we have implemented some existing image encryption methods to compare with our encryption method (see Tables 2 and 3). The entropy, correlation, and differential attack (NPCR and UACI) results and encryption time show the good performance of the proposed image encryption method.

3.3. Sound Steganography Application. In this section, the $128 \times 128$ encrypted picture obtained in Section 3.2 has
TABLE 2: Security analysis result and comparisons $(256 \times 256$ "pepper" image).

\begin{tabular}{lcccc}
\hline & Entropy & Correlation & NPCR & UACI \\
\hline This work & 7.9972 & 0.0042 & 99.9802 & 30.0634 \\
Ref. [52] & 7.9560 & 0.5210 & 99.6289 & 31.8345 \\
Ref. [53] & 7.9972 & 0.0520 & 99.6109 & 12.7548 \\
Ref. [54] & 7.9820 & 0.0052 & 99.5773 & 34.1402 \\
Ref. [55] & 7.9958 & 0.0068 & 99.6170 & 25.125 \\
Ref. [56] & 7.9991 & 0.0082 & 99.028 & 33.10 \\
Ref. [57] & 7.998 & 0.0071 & 99.50 & 33.39 \\
\hline
\end{tabular}

TABLE 3: Encryption time and comparisons $(256 \times 256$ "pepper" image).

\begin{tabular}{lc}
\hline & Encryption time (s) \\
\hline This work & 0.4865 \\
Ref. [52] & 1.6734 \\
Ref. [58] & 3.704 \\
Ref. [59] & 0.712 \\
Ref. [60] & 5.6544 \\
Ref. [61] & 0.5630 \\
\hline
\end{tabular}

been hidden in the original sound as in Figure 9. In this hiding process, firstly, the pixel values of the encrypted picture are converted into binary numbers. Secondly, the float values obtained from the sound data are converted to 32-bit binary numbers. Finally, all the bits belonging to the encrypted image are hidden by placing them in the LSB bits of the 32-bit binary numbers of the selected sound data using the RNG obtained in Section 3.1. As a result, the sound data in which the encrypted image is hidden is shown in Figure 9. There is no visual difference 


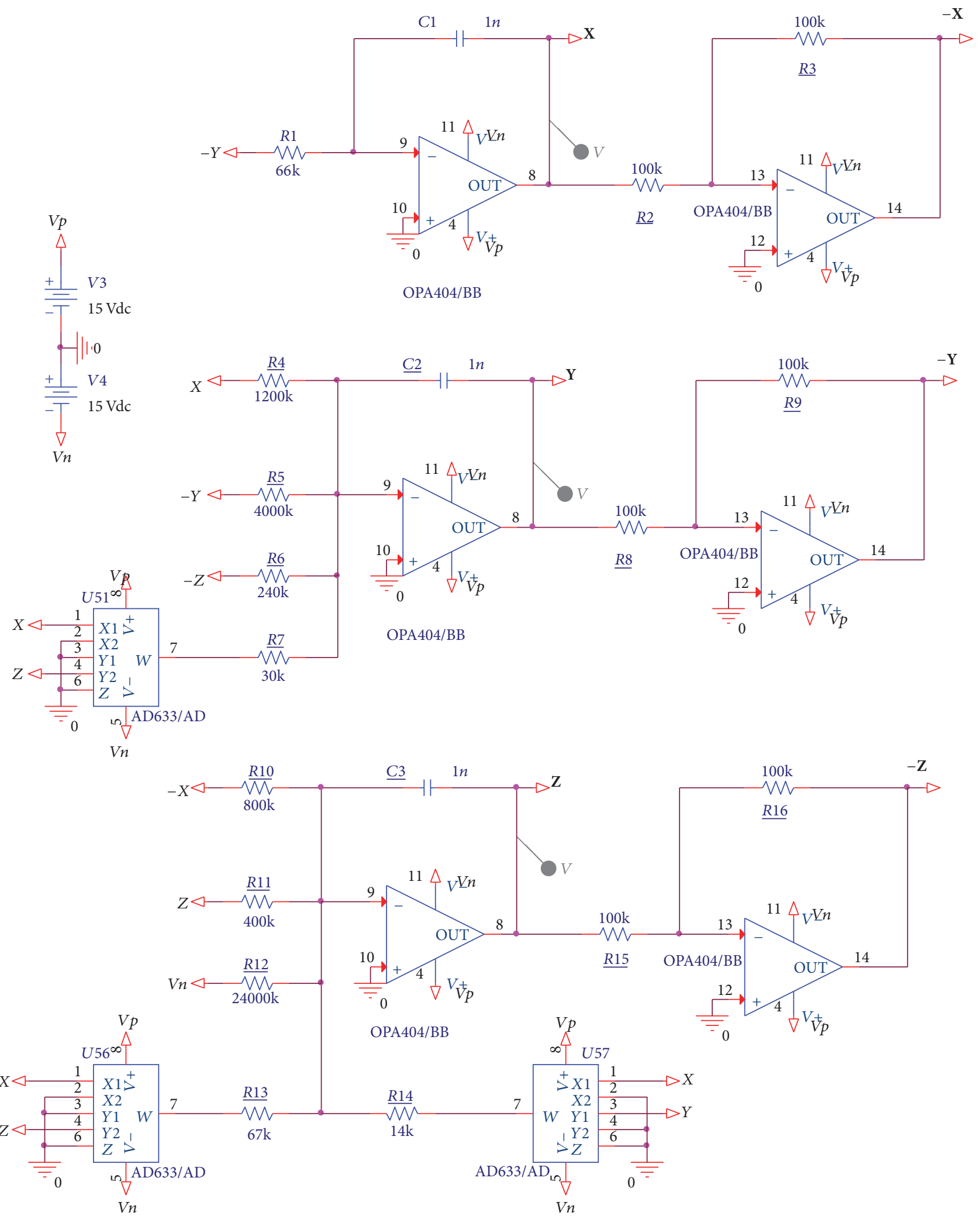

FIGURE 3: The circuit schematic of the scaled system. 


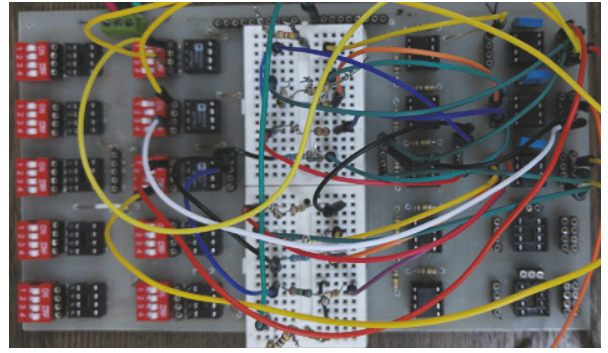

(a)

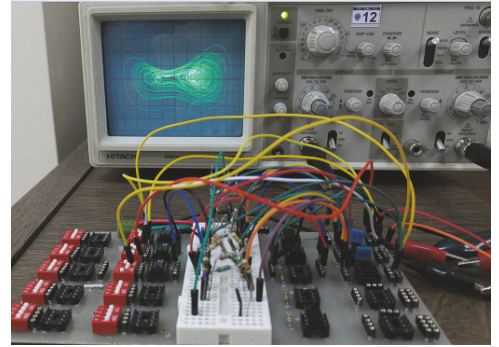

(b)

FIGURE 4: The experimental circuit of the scaled chaotic system.

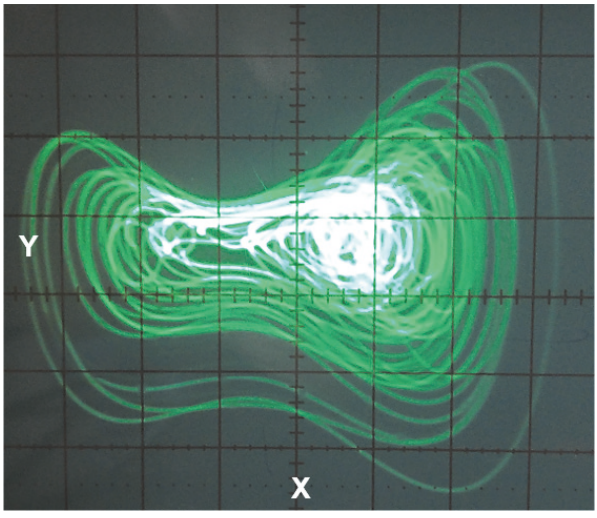

(a)

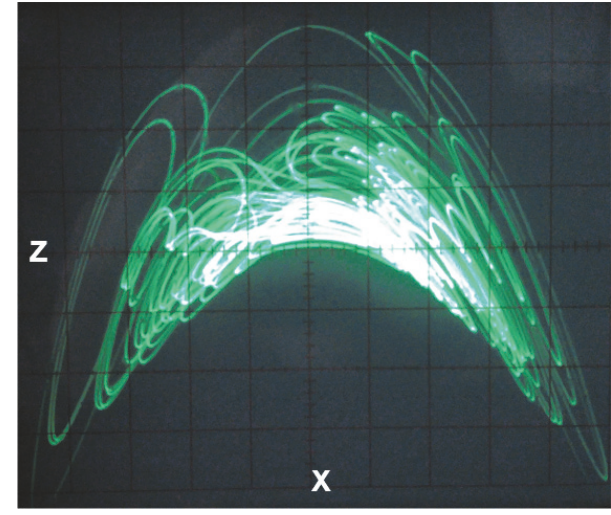

(b)

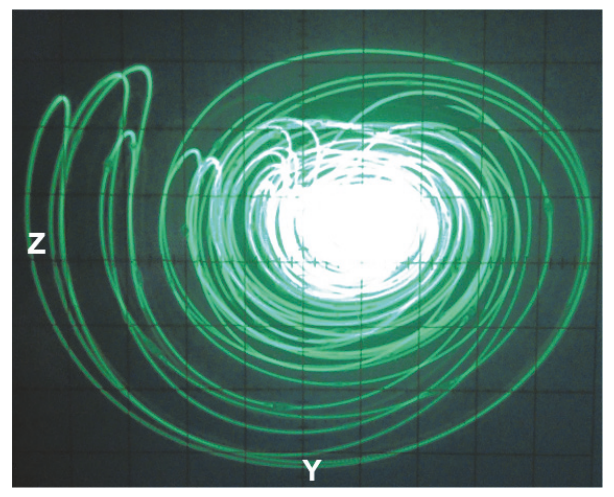

(c)

Figure 5: All phase portraits of the scaled chaotic system on the oscilloscope: (a) $X-Y$ plane, (b) $X-Z$ plane, and (c) $Y-Z$ plane.

between the original and embedded sound data seen in Figure 9. This shows that the steganography application has a good performance. Since it is not enough that there is no difference visually between the original and embedded sounds, analyses of correlation, histogram, entropy, mean square error (MSE), maximum absolute error (MAXERR), and the energy ratio (L2RAT) were also performed. As seen in Figures 10 and 11, correlation distributions and histograms of the original and embedded sounds are the same. In addition, the results of the analyses in Table 4 indicate that there is no difference between the original and embedded sounds. This means that the sound steganography application based on the chaotic system without equilibrium has a good performance.
TABLE 4: Security analysis of the steganography process.

\begin{tabular}{lccc}
\hline Analysis & Original sound & & Embedded sound \\
\hline Correlation & 0.9994 & & 0.9994 \\
Entropy & 13.4926 & & 13.4926 \\
MSE & & 0 & \\
MAXERR & & 0 & \\
L2RAT & & 1 & \\
\hline
\end{tabular}

\section{Conclusions}

This paper introduces a 3D system without equilibrium, which exhibits chaotic behavior. The system includes ten 


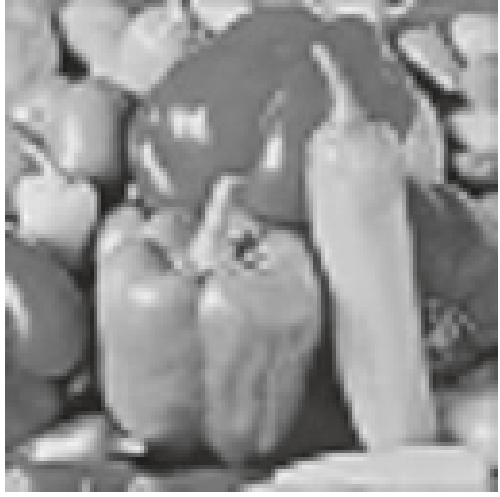

(a)

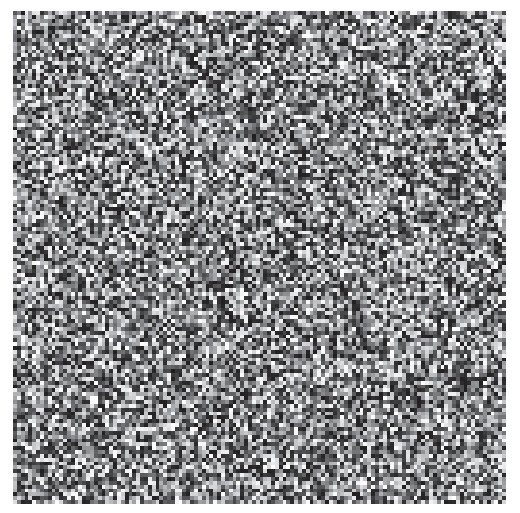

(b)

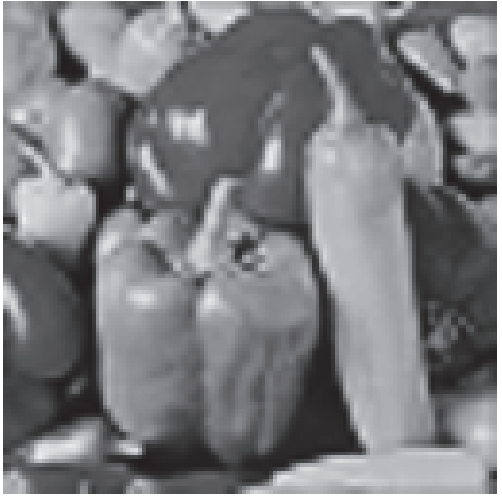

(c)

FIgURE 6: Original, encrypted, and decrypted images.

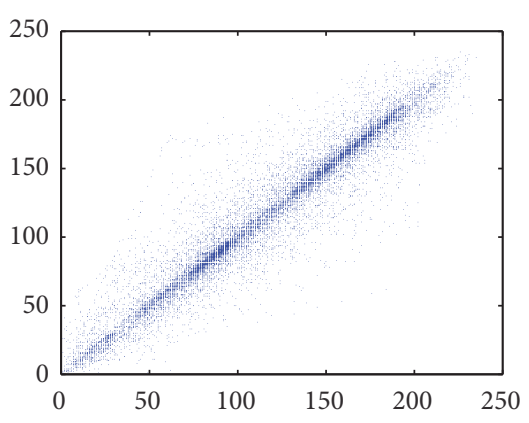

(a)

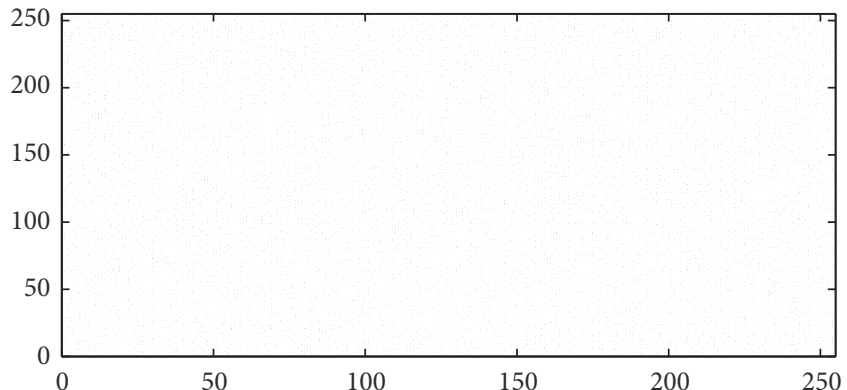

(b)

FIGURE 7: Correlation distributions of original and encrypted images.

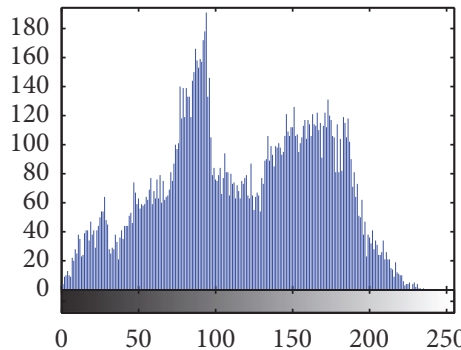

(a)

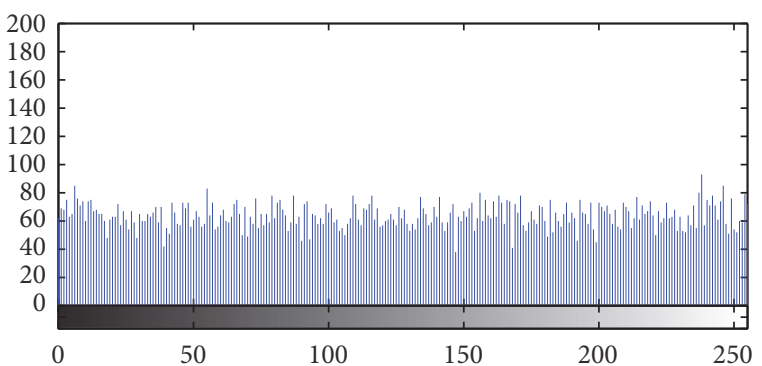

(b)

FIGURE 8: Histograms of original and encrypted images.

terms and has been implemented easily by an electronic circuit. The findings of this work add to a growing list of systems with hidden attractors. This work enhances our understanding of practical applications using systems with hidden attractors. We have used the system without equilibrium for image encryption and sound steganography. According to our knowledge, this is the first time that the $128 \mathrm{kbit}$ data can be encrypted and hidden in sound files. Therefore the findings of this work have important implications for future practice. Other chaotic systems without equilibrium will be discovered in our future researches.

\section{Conflicts of Interest}

The authors declare that there are no conflicts of interest regarding the publication of this paper.

\section{Acknowledgments}

The authors acknowledge Professor GuanRong Chen, Department of Electronic Engineering, City University of Hong Kong, for suggesting many helpful references. The author Xiong Wang was supported by the National Natural 


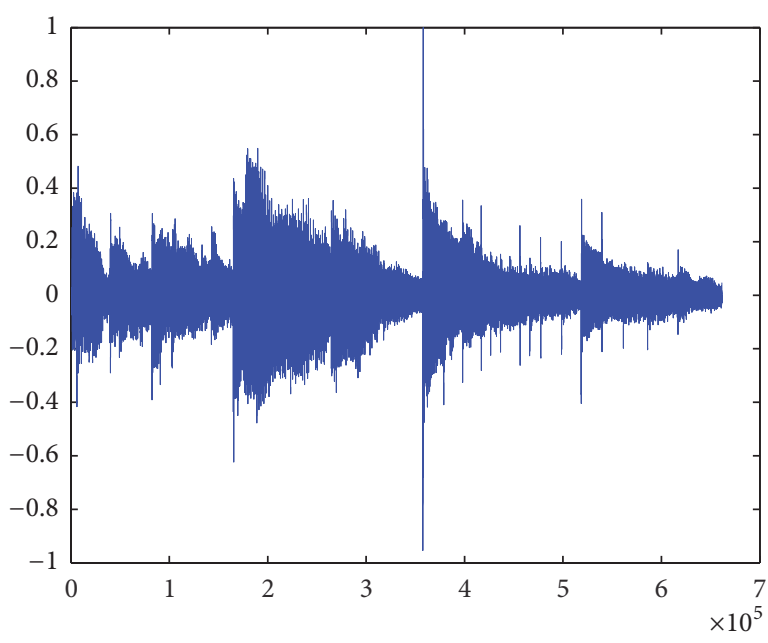

(a)

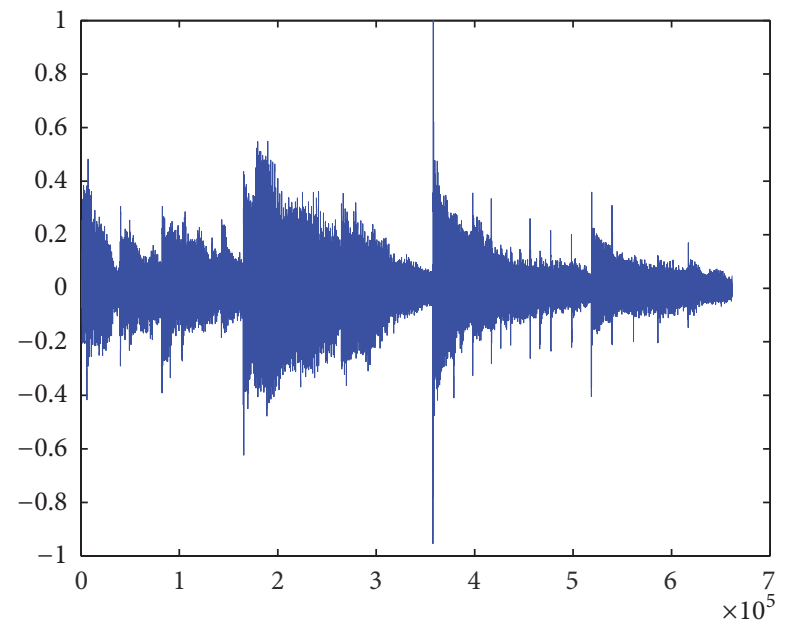

(b)

FIGURE 9: Original and embedded sound.

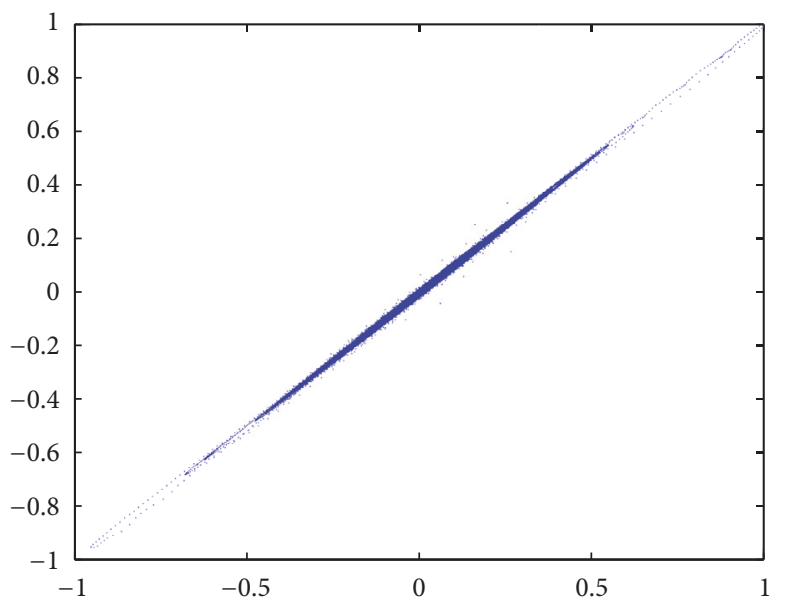

(a)

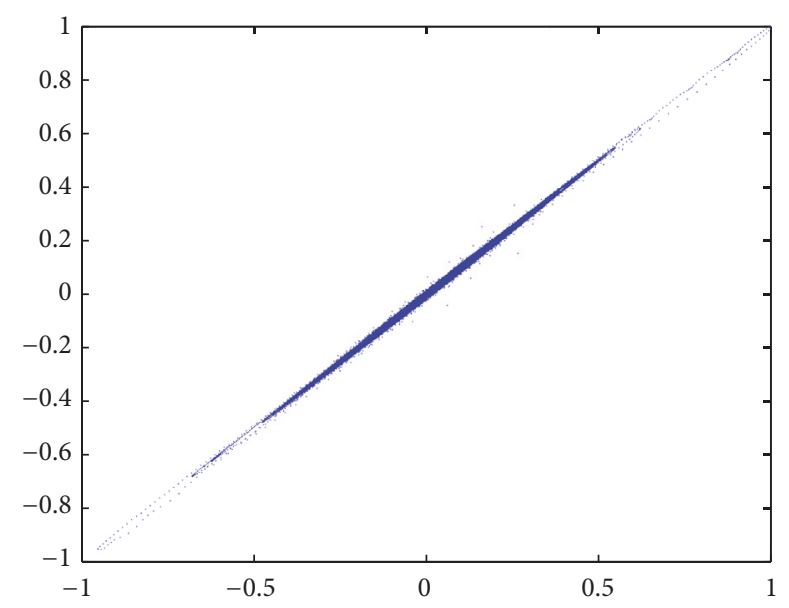

(b)

FIGURE 10: Correlation distributions of original and embedded sounds.

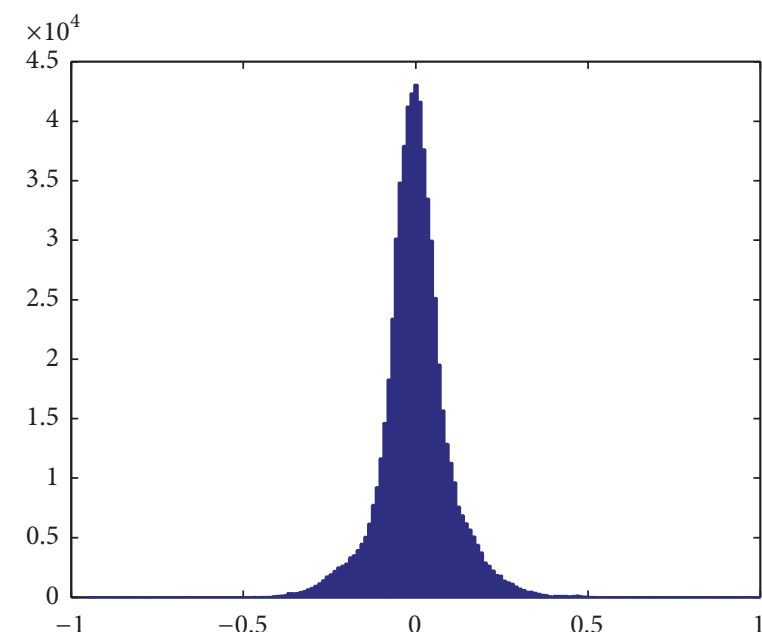

(a)

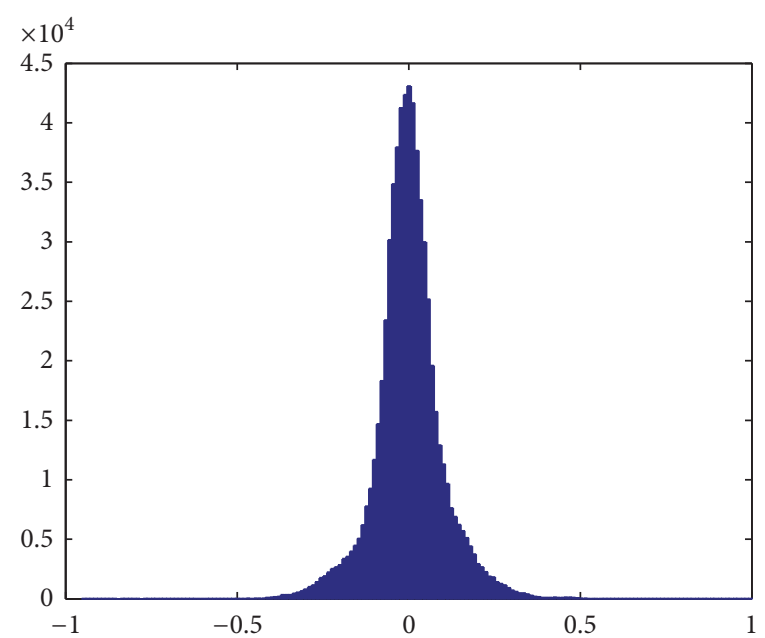

(b)

FIGURE 11: Histograms of original and embedded sounds. 
Science Foundation of China (no. 61601306) and Shenzhen Overseas High Level Talent Peacock Project Fund (no. 20150215145C).

\section{References}

[1] F. Y. Shih, Multimedia Security: Watermaking, Steganography, and Forensics, CRC Press, Boca Raton, Fla, USA, 2013.

[2] A. E. Hassanien, M. M. Fouad, A. A. Manaf, M. Zamani, R. Ahmad, and J. Kacpzyk, Multimedia Forensics and Security Foundations, Innovations, and Applications, Springer, Berlin, Germany, 2017.

[3] N. Dey and V. Santhi, Intelligent Techniques in Signal Processing for Multimedia Security, Springer, Berlin, Germany, 2017.

[4] I. J. Cox, J. Kilian, F. T. Leighton, and T. Shamoon, "Secure spread spectrum watermarking for multimedia," IEEE Transactions on Image Processing, vol. 6, no. 12, pp. 1673-1687, 1997.

[5] M. U. Celik, G. Sharma, E. Saber, and A. M. Tekalp, "Hierarchical watermarking for secure image authentication with localization," IEEE Transactions on Image Processing, vol. 11, no. 6, pp. 585-595, 2002.

[6] J. Eggers and B. Girod, Informed Watermarking, Kluwer Academic, 2002, Kluwer Academic, Dordrecht, The Netherlands, 2002.

[7] M. S. Baptista, "Cryptography with chaos," Physics Letters A, vol. 240, no. 1-2, pp. 50-54, 1998.

[8] K.-W. Wong, "A combined chaotic cryptographic and hashing scheme," Physics Letters. A, vol. 307, no. 5-6, pp. 292-298, 2003.

[9] Z. Dawei, C. Guanrong, and L. Wenbo, "A chaos-based robust wavelet-domain watermarking algorithm," Chaos, Solitons \& Fractals, vol. 22, no. 1, pp. 47-54, 2004.

[10] Y.-T. Wu and F. Y. Shih, "Digital watermarking based on chaotic map and reference register," Pattern Recognition, vol. 40, no. 12, pp. 3753-3763, 2007.

[11] D. Zhao, G. Chen, and W. Liu, "Image scrambling encryption algorithm of pixel bit based on chaos map," Pattern Recognition Letters, vol. 31, no. 5, pp. 347-354, 2010.

[12] G. Alvarez and S. Li, "Some basic cryptographic requirements for chaos-based cryptosystems," International Journal of Bifurcation and Chaos in Applied Sciences and Engineering, vol. 16, no. 8, pp. 2129-2151, 2006.

[13] H. Liu, A. Kadir, and Y. Li, "Audio encryption scheme by confusion and diffusion based on multi-scroll chaotic system and one-time keys," Optik - International Journal for Light and Electron Optics, vol. 127, no. 19, pp. 7431-7438, 2016.

[14] H. Liu, A. Kadir, and Y. Li, "Asymmetric color pathological image encryption scheme based on complex hyper chaotic system," Optik, vol. 127, no. 15, pp. 5812-5819, 2016.

[15] Q. Liu, P.-Y. Li, M.-C. Zhang, Y.-X. Sui, and H.-J. Yang, "A novel image encryption algorithm based on chaos maps with Markov properties," Communications in Nonlinear Science and Numerical Simulation, vol. 20, no. 2, pp. 506-515, 2015.

[16] M. Ghebleh and A. Kanso, "A robust chaotic algorithm for digital image steganography," Communications in Nonlinear Science and Numerical Simulation, vol. 19, no. 6, pp. 1898-1907, 2014.

[17] G. Arumugam, V. Lakshmi Praba, and S. Radhakrishnan, "Study of chaos functions for their suitability in generating Message Authentication Codes," Applied Soft Computing Journal, vol. 7, no. 3, pp. 1064-1071, 2007.
[18] G. A. Leonov, N. V. Kuznetsov, and V. I. Vagaitsev, "Localization of hidden Chua's attractors," Physics Letters A, vol. 375, no. 23, pp. 2230-2233, 2011.

[19] G. A. Leonov, N. V. Kuznetsov, and V. I. Vagaitsev, "Hidden attractor in smooth Chua systems," Physica D: Nonlinear Phenomena, vol. 241, no. 18, pp. 1482-1486, 2012.

[20] G. A. Leonov and N. V. Kuznetsov, "Hidden attractors in dynamical systems: from hidden oscillations in hilbertkolmogorov, Aizerman, and Kalman problems to hidden chaotic attractor in chua circuits," International Journal of Bifurcation and Chaos, vol. 23, no. 1, Article ID 1330002, 2013.

[21] D. Dudkowski, S. Jafari, T. Kapitaniak, N. V. Kuznetsov, G. A. Leonov, and A. Prasad, "Hidden attractors in dynamical systems," Physics Reports, vol. 637, pp. 1-50, 2016.

[22] T. Kapitaniak, "Generating strange nonchaotic trajectories," Physical Review E, vol. 47, no. 2, pp. 1408-1410, 1993.

[23] B. Blazejczyk-Okolewska and T. Kapitaniak, "Dynamics of impact oscillator with dry friction," Chaos, Solitons \& Fractals, vol. 7, no. 9, pp. 1455-1459, 1996.

[24] B. Blazejczyk-Okolewska and T. Kapitaniak, "Co-existing attractors of impact oscillator," Chaos, Solitons \& Fractals, vol. 9, no. 8, pp. 1439-1443, 1998.

[25] S. Kraut and U. Feudel, "Multistability, noise, and attractor hopping: the crucial role of chaotic saddles," Physical Review E: Statistical, Nonlinear, and Soft Matter Physics, vol. 66, no. 1, Article ID 015207, pp. 015207/1-015207/4, 2002.

[26] S. L. T. de Souza, A. M. Batista, I. L. Caldas, R. L. Viana, and T. Kapitaniak, "Noise-induced basin hopping in a vibro-impact system," Chaos, Solitons and Fractals, vol. 32, no. 2, pp. 758-767, 2007.

[27] U. Feudel, "Complex dynamics in multistable systems," International Journal of Bifurcation and Chaos in Applied Sciences and Engineering, vol. 18, no. 6, pp. 1607-1626, 2008.

[28] M. D. Shrimali, A. Prasad, R. Ramaswamy, and U. Feudel, "The nature of attractor basins in multistable systems," International Journal of Bifurcation and Chaos, vol. 18, no. 6, pp. 1675-1688, 2008.

[29] A. Chudzik, P. Perlikowski, A. Stefanski, and T. Kapitaniak, "Multistability and rare attractors in van der Pol-Duffing oscillator," International Journal of Bifurcation and Chaos in Applied Sciences and Engineering, vol. 21, no. 7, pp. 1907-1912, 2011.

[30] A. N. Pisarchik and U. Feudel, "Control of multistability," Physics Reports, vol. 540, no. 4, pp. 167-218, 2014.

[31] G. A. Leonov, N. V. Kuznetsov, M. A. Kiseleva, E. P. Solovyeva, and A. M. Zaretskiy, "Hidden oscillations in mathematical model of drilling system actuated by induction motor with a wound rotor," Nonlinear Dynamics, vol. 77, no. 1-2, pp. 277-288, 2014.

[32] G. A. Leonov, N. V. Kuznetsov, and T. N. Mokaev, "Hidden attractor and homoclinic orbit in Lorenz-like system describing convective fluid motion in rotating cavity," Communications in Nonlinear Science and Numerical Simulation, vol. 28, no. 1-3, pp. 166-174, 2015.

[33] Z. T. Zhusubaliyev, E. Mosekilde, A. N. Churilov, and A. Medvedev, "Multistability and hidden attractors in an impulsive Goodwin oscillator with time delay," European Physical Journal: Special Topics, vol. 224, no. 8, pp. 1519-1539, 2015.

[34] M. A. Kiseleva, N. V. Kuznetsov, and G. A. Leonov, "Hidden attractors in electromechanical systems with and without equilibria," IFAC-PapersOnLine, vol. 49, no. 14, pp. 51-55, 2016. 
[35] H. Jiang, Y. Liu, Z. Wei, and L. Zhang, "Hidden chaotic attractors in a class of two-dimensional maps," Nonlinear Dynamics, vol. 85, no. 4, pp. 2719-2727, 2016.

[36] N. V. Kuznetsov, G. A. Leonov, M. V. Yuldashev, and R. V. Yuldashev, "Hidden attractors in dynamical models of phaselocked loop circuits: limitations of simulation in MATLAB and SPICE," Communications in Nonlinear Science and Numerical Simulation, vol. 51, pp. 39-49, 2017.

[37] M.-F. Danca, N. Kuznetsov, and G. Chen, "Unusual dynamics and hidden attractors of the Rabinovich-Fabrikant system," Nonlinear Dynamics, vol. 88, no. 1, pp. 791-805, 2017.

[38] Z. Wei, "Dynamical behaviors of a chaotic system with no equilibria," Physics Letters. A, vol. 376, no. 2, pp. 102-108, 2011.

[39] Z. Wang, S. Cang, E. O. Ochola, and Y. Sun, "A hyperchaotic system without equilibrium," Nonlinear Dynamics, vol. 69, no. 1-2, pp. 531-537, 2012.

[40] S. Jafari, J. C. Sprott, and S. . Hashemi Golpayegani, "Elementary quadratic chaotic flows with no equilibria," Physics Letters A, vol. 377, no. 9, pp. 699-702, 2013.

[41] X. Wang and G. Chen, "Constructing a chaotic system with any number of equilibria," Nonlinear Dynamics, vol. 71, no. 3, pp. 429-436, 2013.

[42] Z. Wei, R. Wang, and A. Liu, "A new finding of the existence of hidden hyperchaotic attractors with no equilibria," Mathematics and Computers in Simulation, vol. 100, pp. 13-23, 2014.

[43] C. K. Volos, I. M. Kyprianidis, and I. N. Stouboulos, "A chaotic path planning generator for autonomous mobile robots," Robotics and Autonomous Systems, vol. 60, no. 4, pp. 651-656, 2012.

[44] S. Bouali, A. Buscarino, L. Fortuna, M. Frasca, and L. V. Gambuzza, "Emulating complex business cycles by using an electronic analogue," Nonlinear Analysis: Real World Applications, vol. 13, no. 6, pp. 2459-2465, 2012.

[45] C. K. Volos, I. M. Kyprianidis, and I. N. Stouboulos, "Image encryption process based on chaotic synchronization phenomena," Signal Processing, vol. 93, no. 5, pp. 1328-1340, 2013.

[46] A. Buscarino, L. Fortuna, M. Frasca, and G. Sciuto, "Design of time-delay chaotic electronic circuits," IEEE Transactions on Circuits and Systems I: Regular Papers, vol. 58, no. 8, pp. 18881896, 2011.

[47] W.-J. Zhou, Z.-P. Wang, M.-W. Wu, W.-H. Zheng, and J.-F. Weng, "Dynamics analysis and circuit implementation of a new three-dimensional chaotic system," Optik, vol. 126, no. 7-8, Article ID 55317, pp. 765-768, 2015.

[48] Q. Lai and L. Wang, "Chaos, bifurcation, coexisting attractors and circuit design of a three-dimensional continuous autonomous system," Optik, vol. 127 , no. 13 , pp. 5400-5406, 2016.

[49] A. Akgul, I. Moroz, I. Pehlivan, and S. Vaidyanathan, "A new four-scroll chaotic attractor and its engineering applications," Optik - International Journal for Light and Electron Optics, vol. 127, no. 13, pp. 5491-5499, 2016.

[50] A. Akgul, S. Hussain, and I. Pehlivan, "A new three-dimensional chaotic system, its dynamical analysis and electronic circuit applications," Optik, vol. 127, no. 18, pp. 7062-7071, 2016.

[51] B. Bao, T. Jiang, Q. Xu, M. Chen, H. Wu, and Y. Hu, "Coexisting infinitely many attractors in active band-pass filter-based memristive circuit," Nonlinear Dynamics, vol. 86, no. 3, pp. 1711-1723, 2016.

[52] Ü. Çavuşoğlu, S. Kaçar, I. Pehlivan, and A. Zengin, "Secure image encryption algorithm design using a novel chaos based S-Box," Chaos, Solitons and Fractals, vol. 95, pp. 92-101, 2017.
[53] O. M. Al-Hazaimeh, N. Alhindawi, S. M. A. Hayajneh, and A. Almomani, "HANON chaotic map - based new digital image encryption algorithm," MAGNT Research Report, vol. 2, pp. 261266, 2014.

[54] H. M. Al-Naijar, "Digital image encryption algorithm based on a linear independence scheme and the logistic map," in Proceedings of the International Arab Conference on Information Technology (ACIT '11), pp. 215-219, 2011.

[55] H. Al-Najjar and N. Al-Rousan, "Data hiding in encrypted image based on multi-chaotic approach," International Journal of Scientific and Engineering Research, vol. 4, pp. 1-5, 2013.

[56] R. Enayatifar, A. H. Abdullah, and I. F. Isnin, "Chaos-based image encryption using a hybrid genetic algorithm and a DNA sequence," Optics and Lasers in Engineering, vol. 56, no. 5, pp. 83-93, 2014.

[57] R. Enayatifar, H. J. Sadaei, A. H. Abdullah, M. Lee, and I. F. Isnin, "A novel chaotic based image encryption using a hybrid model of deoxyribonucleic acid and cellular automata," Optics and Lasers in Engineering, vol. 71, pp. 33-41, 2015.

[58] S. Lian, J. Sun, and Z. Wang, "A block cipher based on a suitable use of the chaotic standard map," Chaos, Solitons \& Fractals, vol. 26, no. 1, pp. 117-129, 2005.

[59] K. Wong, B. S. Kwok, and W. Law, "A fast image encryption scheme based on chaotic standard map," Physics Letters, Section A: General, Atomic and Solid State Physics, vol. 372, no. 15, pp. 2645-2652, 2008.

[60] Y. Wu, G. Yang, H. Jin, and J. P. Noonan, "Image encryption using the two-dimensional logistic chaotic map," Journal of Electronic Imaging, vol. 21, no. 1, Article ID 013014, 2012.

[61] X. Liao, S. Lai, and Q. Zhou, "A novel image encryption algorithm based on self-adaptive wave transmission," Signal Processing, vol. 90, no. 9, pp. 2714-2722, 2010. 


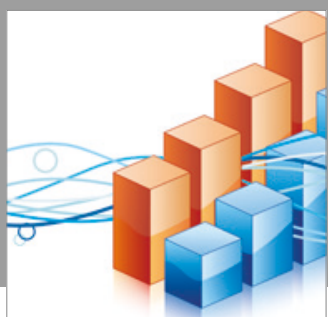

Advances in

Operations Research

vatersals

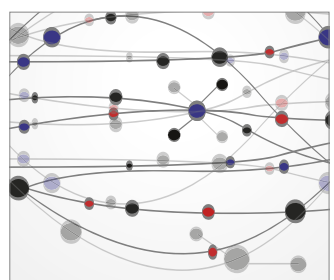

\section{The Scientific} World Journal
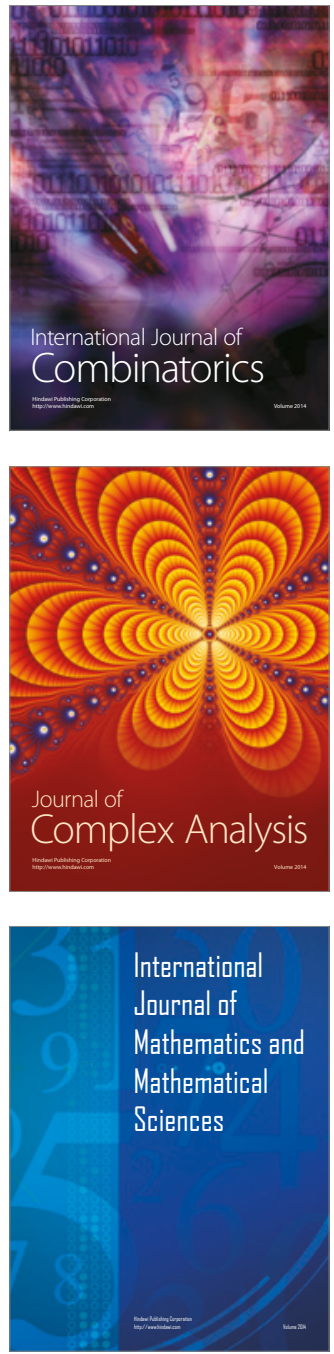
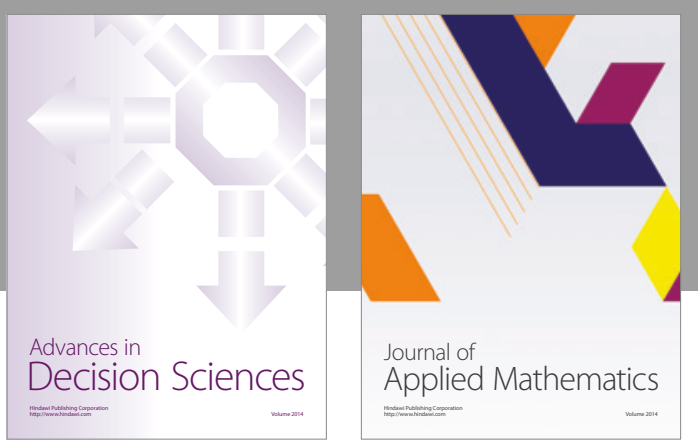

Algebra

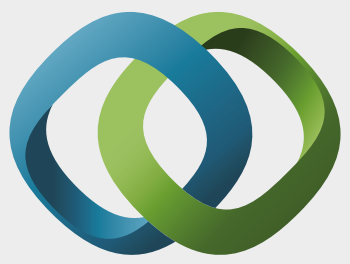

\section{Hindawi}

Submit your manuscripts at

https://www.hindawi.com
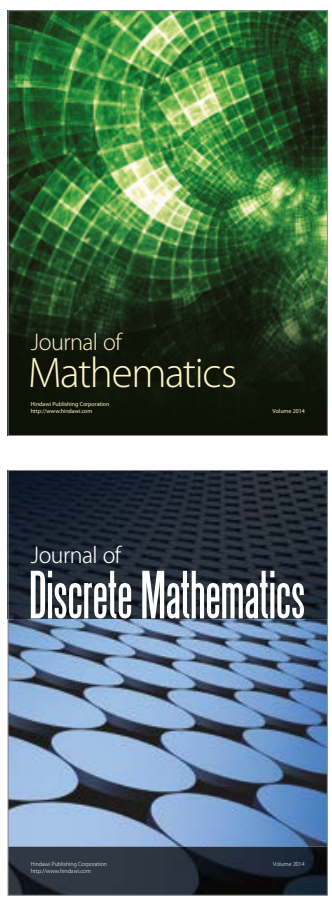

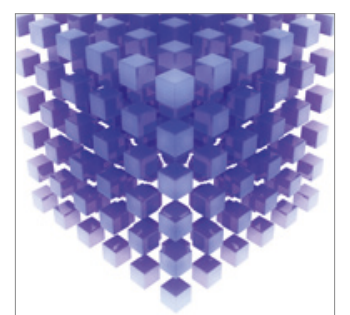

Mathematical Problems in Engineering
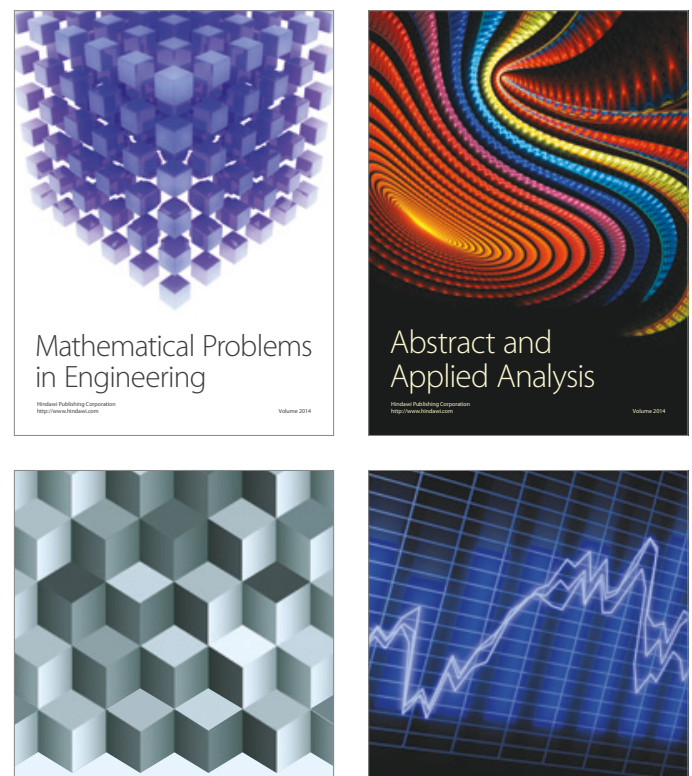

Journal of

Function Spaces

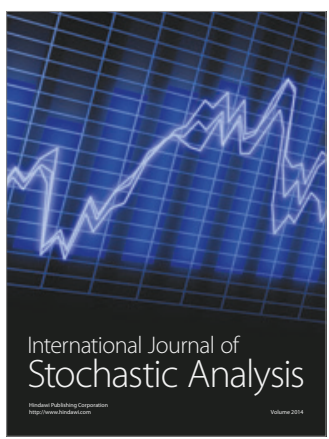

Probability and Statistics
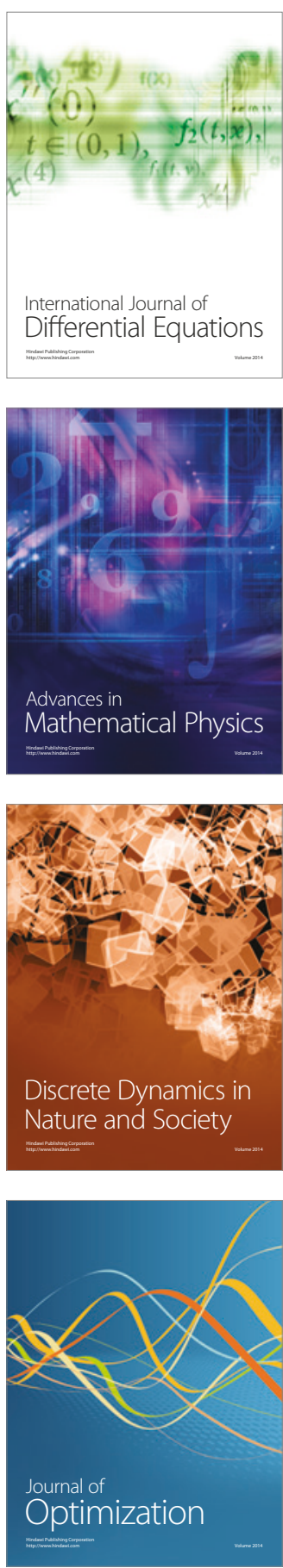